\title{
Comparison of different error signals driving the adaptation in assist-as-needed controllers for neurorehabilitation with an upper-limb robotic exoskeleton
}

\author{
Tommaso Proietti, Guillaume Morel, Agnès Roby-Brami, and Nathanaël Jarrassé
}

\begin{abstract}
Assist-as-needed control aims at maximizing stroke survivors involvement during robotic-led therapies of neurorehabilitation. Besides the specific characteristics of the designed adaptive control strategy, a fundamental property of this control architecture is the choice of the error signal which will drive the adaptation process. This driving source is a necessary control parameter to be chosen, although often sidelined in the control design, and several solutions already exist in the state-of-the-art. For this reason, we wanted to compare three different strategies to guide the adaptation, respectively based on the local joint performances, on the endeffector only behaviour, or on the performance of one specific joint in the kinematic chain of the robot. The resulting analysis evaluates the possibilities offered by simply changing from one source to another with respect to the specific stage of the motor recovery of the patients, potentially extending the capabilities of current exoskeleton controllers for neurorehabilitation.
\end{abstract}

\section{INTRODUCTION}

Stroke is one of the main causes of acquired neurological impairments in adults worldwide. Stroke survivors are usually left with disability, mainly motor impairments on both upper- and lower-limb movements and loss of hand dexterity, which are partially recoverable by undergoing rehabilitation [1]. Neurorehabilitation should provide intense and challenging physical training to the patients, progressing together with their capabilities, and the therapy should engage patients in active participations [2].

Rehabilitation robotics has been studied and developed for about twenty-five years because of its interesting features for clinical applications [3]. Robots are, in fact, capable of ensuring repetitive and intense therapeutic sessions, they can provide reliable quantitative measurements of the patient performance during the therapy, and thanks to virtual environment, these devices can increase subject's motivation by producing up-to-date informations on the performance, challenging the patients with involving games and providing scores [4].

In the literature, the most common approach for controlling exoskeletons for neurorehabilitation involves passive control [5] modes: the subject is passive while the robot performs the motion, mostly through a stiff impedance control. However, the effectiveness of such passive motions for stimulating motor recovery is limited [6]. Assistive control modes

Authors are with Sorbonne Universités, UPMC Univ. Paris 06, CNRS, AGATHE U1150, Institut National de la Santé et de la Recherche Médicale (INSERM), UMR 7222, the Institute of Intelligent Systems and Robotics (ISIR), 4 place Jussieu, 75005, Paris, France. \{proietti, morel, roby-brami, jarrasse\}@isir .upmc . fr shall thus be used, to let the subject actively control the motion. In this case, the robot provides assistance by virtually constraining the motion and by supporting the impaired limb, based on performance indexes, as for example in [7] or in [8]. Ultimately, it is essential for rehabilitation robots to allow a shared control of the movements, as soon as the patient has recovered a minimal amount of motor capacity [9], [10]. Indeed, as neurorehabilitation addresses issues related to motor control relearning, the devices must allow patients to express whatever natural, healthy-like movement they can, without suppressing any motor capability [11].

In this sense, techniques from adaptive control may lead to improvements: adaptation can allow the robot to modulate its assistance level based on the subject's motor conditions. Advantageously, adaptation can also tune the controller to account the differences between patients.

Thus Assist-As-Needed (AAN) controllers have been developed in order to maximize patients involvement by minimizing robot necessary intervention. There exist different implementations to produce AAN, but these usually involve a feedforward assistance in addition to a feedback controller, and the adaptation is generally run offline, trial-bytrial. The adaptation occur either at the feedback level (PD gains adaptation to modify the compliance of the robot, with a fixed forgetting factor to reduce assistance [12], or with an adaptive forgetting factor [13], or with sensorless force estimation in order to model subject's capabilities and avoiding the forgetting term [14]) or at the feedforward level (adaptation of the feedforward term to address repetitive consistent errors [15], or with an additional neural network to model the subject capabilities [16]).

Whatever the solution adopted to provide AAN, limited attention has been yet given to the source of the adaptation, that is the error signal reflecting the performance of the operator and driving the adaptation process. In a tracking task, for example, adapting the behaviour of the robotic exoskeleton only based on the end-effector performance, rather than utilizing multiple different joint-based indexes, can imply sufficient different adaptations and thus robot performances. End-effector based adaptation can be the solution when functional recovery is needed (regaining of independence and functionality for performing activities of daily living), but for impairment recovery, thus addressing regaining of strength and muscle tone, range of motion, as well as joints arm coordination, for example to avoid negative trunk compensation while extending the arm, a joint-level 
adaptation could be more effective. It is then clear that, in order to correctly use AAN in rehabilitation therapy, with neurological disease survivors, a specific analysis should be done to determine what possible sources of adaptation exist and which potentiality each of them could exhibit.

In this paper, we study three different error signals driving the adaptation process in an AAN controller, in order to better determine which one should be used with respect to the specific aspect of the rehabilitation process that is targeted during the recovery of the patient. To this aim we utilize the above-mentioned controller we developed for a former research on AAN [12], even if we believe our results could be directly applied to most of the existing control architectures for rehabilitation robotics, for example with impedance controllers which are one of the most common solutions [5].

\section{Assist-As-Needed Control}

An AAN controller aims at maximizing subject participation, by finding the minimum necessary level of assistance to complete the desired task. Our AAN control strategy produces an output $\mathbf{w} \in \mathbb{R}^{n}$, where $n$ is the number of joints of the robotic exoskeleton, which is composed of two terms

$$
\mathbf{w}=\mathbf{u}+\mathbf{v}
$$

where $\mathbf{u} \in \mathbb{R}^{n}$ is a feedforward term, and $\mathbf{v} \in \mathbb{R}^{n}$ is a feedback control. In particular, the feedforward torque is a modelbased gravity compensation, which helps the subject not to feel the weight of the robot while inside the exoskeleton. The feedback $\mathbf{v}$, instead, is an adaptive proportional-derivative (PD) control, thus similar to an impedance control without the inertial term:

$$
\mathbf{v}=\mathbf{K}_{p} \mathbf{e}+\mathbf{K}_{d} \dot{\mathbf{e}}, \quad \mathbf{e}=\mathbf{q}_{r}(t)-\mathbf{q}(t)
$$

where $\mathbf{q}(t)$ is the joint position vector, and error signals e and $\dot{\mathbf{e}}$ are calculated with respect to the reference joint trajectory $\mathbf{q}_{r}(t)$ and the reference joint velocity $\dot{\mathbf{q}}_{r}(t) . \mathbf{K}_{p}$, the stiffness term, and $\mathbf{K}_{d}$, the damping term, are positive diagonal matrices of gains.

Through this simple control scheme, the robot imposes the reference trajectory $\mathbf{q}_{r}(t)$ to the subject's joints when the stiffness is high (large values of $\mathbf{K}_{p}$ and $\mathbf{K}_{d}$ ), which is suited for early rehabilitation exercises, when the subject cannot provide large forces. In this case, the robot torques are large and the subject is mostly passive in the exoskeleton. During the recovery, as soon as relearning is occurring, the subject is expected to be able to provide more energy, in order to complete the task more autonomously, and thus the robot needs to decrease its assistance. In our control, this is achieved by decreasing the stiffness of the robotic arm (small values of $\mathbf{K}_{p}$ and $\mathbf{K}_{d}$ ). At the lowest limit, i.e. when null $\mathbf{K}_{p}$ and $\mathbf{K}_{d}$ are reached, the robot is only compensated for the gravity by the $\mathbf{u}$ feedforward term, thus leaving the exoskeleton in a transparent mode (no assistance/resistance to the free motion of the subject), and the subject completely active and free to perform any movement.

\section{Adaptation law}

To achieve this adaptive behaviour, the gains of the PD controller are adapted trial-by-trial, based on the former performance of the subject, similarly to what is proposed by Ganesh et al [17] for achieving adaptive haptic exploration. If the subject is correctly performing the requested task, the robot should try to reduce its assistance, and vice versa if the performance is not satisfying, the robot should rather increase its assistance. Namely, at a given trial $k$, the controller proportional gain $K_{p, i}$ for the $i$-th joint of the exoskeleton is computed by:

$$
K_{p, i}^{k}=K_{p, i}^{k-1}+\beta_{i} z_{i}^{k-1}-\gamma_{i}
$$

where the learning parameter $\beta_{i}$ and the decay $\gamma_{i}$ are positive scalars, and $z_{i}$ represents the reference parameter at trial $k-1$ to evaluate the performance of the $i$-th joint. In addition, to avoid large increasing of the robotic stiffness and thus awkward feelings on the arm of the human operator, the $\Delta K_{p, i}=\beta_{i} z_{i}^{k-1}-\gamma_{i}$ is upper-saturated such that $\max \left(\Delta K_{p, i}\right)=$ $\gamma_{i}$. At the same time the damping $K_{d, i}$ varies with the proportional gain

$$
K_{d, i}^{k}=\alpha_{i} K_{p, i}^{k} .
$$

Both gains are saturated between $K_{\max }$ and $K_{\min }>0$, with

$$
\alpha_{i}=\frac{K_{d, i}^{\max }-K_{d, i}^{\min }}{K_{p, i}^{\max }-K_{p, i}^{\min }}\left(K_{p, i}-K_{p, i}^{\min }\right)+K_{d, i}^{\min } .
$$

\section{Error signals choice}

We defined three different error signals capable of driving the adaptation process of equation 3: end-effector performance based error signal, joint-by-joint performance based error signal, and single-joint performance based error signal.

1) End-Effector Based adaptation (EEB): One intuitive solution to drive the adaptation is by comparing, trial-bytrial, the performance of the end-effector and the desired behaviour. The error computed at the end-effector (either position, velocity or both position and velocity error ${ }^{1}$ ) is then globally modifying the behaviour of the robot, that is, all the robotic joints are adapting their control laws with the same ratio. With our controller, the EEB solution is given by defining the $z_{i}$ as

$$
z_{i} \triangleq z=\sum_{t=0}^{T}\left(\left\|\mathbf{e}_{x}\right\|+\xi\left\|\dot{\mathbf{e}}_{x}\right\|\right) \quad i \in[1 \ldots n]
$$

where $\xi$ is a scaling factor between the position and the velocity error, $\left\|\mathbf{e}_{x}\right\|=\left\|\mathbf{p}_{\text {des }}-\mathbf{p}_{e e}\right\|=$ $\sqrt{\left(x_{d e s}-x_{e e}\right)^{2}+\left(y_{d e s}-y_{e e}\right)^{2}+\left(z_{d e s}-z_{e e}\right)^{2}}$ is the end-effector position error, where we removed the dependence of time (e.g. $\left.x_{d e s}(t)\right)$ for a matter of readability, and similarly for the end-effector velocity error $\left\|\dot{\mathbf{e}}_{x}\right\|=\left\|\dot{\mathbf{p}}_{e e}-\dot{\mathbf{p}}_{d e s}\right\|$.

\footnotetext{
${ }^{1}$ Since AAN controllers usually address early post-stroke therapies, faster correct movements than the desired one are unlikely to happen. The robot should thus take care of the speed of the motion, stopping too fast or too slow, probably unnatural, movements. When considering more advanced therapies, on the other side, the constraint on the velocity could decrease the healthy freedom of the patients, and thus should be removed.
} 
2) Joint-By-Joint based adaptation (JBJ): In JBJ, the error signal in equation 3 is locally computed for each joint. In fact, the error takes into account the single joint performance to adapt its stiffness. Therefore, with this strategy, each joint may evolve differently. The JBJ performance index $z_{i}$, for the $i$-th joint, is given by

$$
z_{i}=\sum_{t=0}^{T}\left(\left|e_{i}\right|+\xi\left|\dot{e}_{i}\right|\right) \quad i \in[1 \ldots n]
$$

where $\left|e_{i}\right|=\left|\theta_{i}^{\text {des }}(t)-\theta_{i}(t)\right|$ is the joint position error norm and $\left|\dot{e}_{i}\right|=\left|\dot{\theta}_{i}^{\text {des }}(t)-\dot{\theta}_{i}(t)\right|$ is the joint velocity error norm, both for the $i$-th joint.

3) Single-Joint Based adaptation (SJB): In SJB, the performance of one specific joint drives the adaptation of the whole exoskeleton. Therefore, similarly to EEB, the SJB globally modifies the compliance of the robotic arm by considering the same error for the different joints. We defined the driving variable as the flexion/extension of the elbow angle $\alpha_{H F}$, described by the International Society of Biomechanics [18], that is the angle between the upper-arm and the forearm. The coordination between these shoulder and elbow is often impaired in post-stroke survivors [19], and thus focusing on the resulting angle at the elbow could be interesting for the therapy. The error on this angle is computed as

$$
z_{i} \triangleq z=\sum_{t=0}^{T}\left(\left|e_{\alpha}\right|+\xi\left|\dot{e}_{\alpha}\right|\right) \quad i \in[1 \ldots n]
$$

where $\left|e_{\alpha}\right|=\left|\alpha_{H F}^{\text {des }}(t)-\alpha_{H F}(t)\right|$ is the the $\alpha_{H F}$ angle error, and $\left|\dot{e}_{\alpha}\right|=\left|\dot{\alpha}_{H F}^{d e s}(t)-\dot{\alpha}_{H F}(t)\right|$ is the $\alpha_{H F}$ angular velocity error.

\section{Materials AND Methods}

\section{A. The ABLE exoskeleton}

The adaptive algorithms were coded on the real time controller (RTLinux, running control loop at $1 \mathrm{kHz}$ ) of an ABLE exoskeleton designed by the CEA-LIST [20], a four active degrees of freedom (DOF) robot, with 3-DOF for the shoulder (for abduction/adduction, internal/external rotation, and flexion/extension) and one for the elbow (for flexion/extension), see figure 1. ABLE has interesting features for robotics rehabilitation, that are a large workspace (it allows $110^{\circ}$ of rotation at the first three axes, and about $130^{\circ}$ at the elbow), a force/torque range compatible with human ones $(18 \mathrm{Nm}$ available on the first two joints, $13 \mathrm{Nm}$ on the last two, producing an equivalent maximum force at the hand of $50 \mathrm{~N}$ ), and above all high backdriveability, thanks to a patented screw-cable mechanical transmission together with a model-based gravity compensation, providing a transparent behaviour, with the robot that can be easily moved without detecting any motion intention of the human operator.

\section{B. Experimental protocol}

In this paper we want to compare the effect of different error signals which can drive the adaptation law in AAN controllers for neurorehabilitation. To determine the capabilities of each adaptive paradigm developed in section II, we used the following protocol: we asked five healthy subjects (aged $24.4 \pm 0.4)$ to perform once the tracking of the outline of a $25 \times 25 \times 4 \mathrm{~cm}^{3}$ foam-made parallelepiped, while seating on a

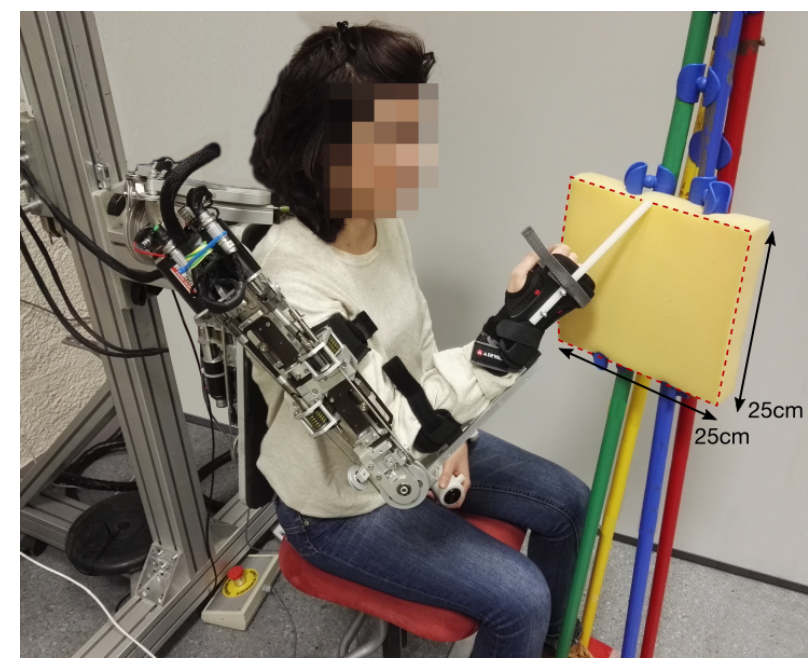

Fig. 1. Experimental setup and ABLE close-up. The subject was told to track the outline of a foam-made parallelepiped with a plastic rod.

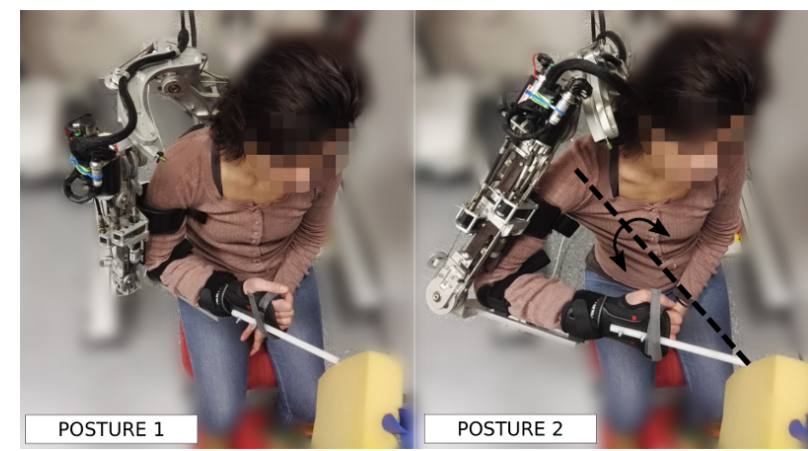

Fig. 2. An example of self-motion: for a same end-effector position, the intra-joint coordination can be voluntary modified thanks to the system redundancy w.r.t. specific tracking task. This different coordination can be achieved and performed during the whole task, producing distinct joint trajectories.

stool. The exoskeleton was connected to the right arm of the subjects through three velcro cuffs, one on the upper-arm and two on the forearm. Besides, the subjects wore a commercial wrist splint to limit wrist motion and prono-supination, not controlled and not measured by the robot, see figure 1 .

To compute the reference trajectories to control the robot, we recorded the joint positions when the subject was pointing at the corners of the squared foam with the exoskeleton in transparency, and then we interpolated straight lines in between them to reproduce the exact contour of the tracked object.

During the experiment, we performed 40 repetitions of the task for each protocol, thus a total of 120 motions, and each motion lasted 10 seconds. By triggering a push-button with the left hand, the subject voluntarily started the motion and the trial recording.

Similarly to the preliminary experiment in [12], we asked the subjects for specific behaviours. In particular we can describe four different phases of the experiment:

- Trial 1 to 25 the subjects were asked to perform the task with the support of the AAN control, 
- Trial 26 to 30 the subjects were asked to relax and let the robot perform the task,

- Trial 31 to 35 the subjects were asked again to perform the task with the support of the AAN control,

- Trial 36 to 40 the subjects were asked to perform the task with the support of the AAN control, but producing a different inter-joint motion coordination.

In the first three phases we expected to see similar behaviours for the three cases of section II, with the robot adapting to the activity of the operator (the simple task should be easily achieved, thus the robot should decrease its assistance in phase 1 and 3, while it increases the stiffness in phase $2)$. We expected small differences in the adaptation strategies, apart from the structural distinction comparing global (EEB and SJB) and local adaptation processes (JBJ). In the fourth phase, instead, when asking to change voluntarily the motion coordination, while keeping the end-effector task achievement, we expected to obtain slightly different gains evolutions due to the variation on the driving error signals.

It is important to underline that we were able to perform the fourth phase exercise thanks to the redundancy of the ABLE exoskeleton (a 4-DOF robot) with respect to the specific task (pointing in the space requires only 3-DOF). In this scenario, in fact, similarly to any pointing task with healthy human arm, there are more available DOF than the required ones and we can achieve the same task (following the squared outline) by performing different strategies at the joint level (usually named as self-motions, see figure 2).

For this experiment, the initial gains were set to $K_{p, i}^{t_{0}}=200$ and $K_{d, i}^{t_{0}}=6.6 \forall i$, and the gain saturations were $K_{p, i}^{\max }=300$, $K_{p, i}^{\min }=0, K_{d, i}^{\max }=10$, and $K_{d, i}^{\min }=0$ for all the joints. In addition, at the end of trial 35 , we set $K_{p, i}=K_{d, i}=0$ at each joint, in order to compare the behaviour of the controllers with the same starting gain configuration (full transparency). $\xi=0.1$ was also set to give more importance to the path tracking, more than to the exact speed profile reproduction.

The tuning of the adaptive parameters $\beta$ and $\gamma$ was a crucial definition. We wanted the robot to be transparent towards the end of the first phase (within 25 movements). For this reason, given $K_{p, i}^{t_{0}}=200$, we selected $\gamma_{i}=8, \forall i$. At the same time, we needed the robot to be able to react sufficiently quick (within 5 trials) to increases of the tracking error. To determine a useful $\beta$ to reproduce this phenomenon, we recorded the error range (joint error for JBJ, $\alpha_{H F}$ error for $\mathrm{SJB}$, and end-effector error for EEB, as defined in section II) when the robot was performing the task alone, without any operator, respectively in rigid mode $\left(\forall K_{p}=300\right.$ and $\forall K_{d}=$ 10) and in transparency $\left(\forall K_{p}=K_{d}=0\right)$. Trivially the rigid mode gave us the minimum error for each protocol, while the transparency the maximum one (indeed the robot did not move during this test). Based on these error ranges and the selected $\gamma$, we computed the values of $\beta$ for each protocol, which were: $\beta_{i, J B J}=\left[\begin{array}{llll}0.1 & 0.1 & 0.05 & 0.05\end{array}\right], \beta_{i, S J B}=0.01$, and $\beta_{i, E E B}=0.01$ for any $i \in[1, \ldots 4]$. The different $\beta$ for the JBJ case were due to different error ranges at the joint 3 and 4 compared to the other joints, for this specific task.

\section{Results}

\section{Trials 1-35: adaptation to the subjects performance}

Figure 3 shows the average evolution of the proportional gain $K_{p, i}$ over the 40 movements and the five subjects, when using the three different error signals. At the same time, figure 4 shows the average errors at the joints and at the endeffector at each trial, for the five subjects. The $K_{p}$ value can be considered proportional to the stiffness of the robotic arm. As expected, all the AAN controllers adapted correctly to the behaviour of the exoskeleton operators. During the first phase (trials 1-25), while the subjects were actively performing the task and consequently the errors were low, the robotic exoskeleton generally became more compliant (decreasing $K_{p}$ ). It is important to see the difference between JBJ local adaptation, in which the four joints adapted differently based on local performances, and the other protocols, in which the performance index was one for all the joints and therefore the gains evolved exactly the same for the whole robotic device. This different behaviour reflected into a larger mean error at each joint, during this interval.

Once the subjects relaxed (trials 26-30), the three controllers correctly increased the stiffness of the robotic arm producing better assistance to the movements (increasing $K_{p}$ ). In this interval, both joint error and end-effector error increased because of the voluntary relaxation of the subject. As soon as relaxation was over, the subjects were again contributing to the performance, the errors were drastically decreasing, and thus the robot compliance increased again.

\section{Trials 36-40: correcting a different coordination}

For the second part of the experiment, in the interval of trials 36-40, we asked the subjects to try to perform the same end-effector task while adopting a different interjoint coordination. The task was performed by increasing both the abduction and the internal rotation of the shoulder with respect to the desired joint trajectories, exploiting the ABLE/human system redundancy (figure 2). Figure 5 shows the resulting behaviour of the robot, for a typical subject. In particular, the joint trajectory of trial 36 (dashed line) and 40 (solid line) are shown for each choice of error signal.

As we can see, the different inter-joint coordination was "allowed" by the EEB adaptation, in which clearly the performance index (the end-effector error, which remained small) was not influenced by joint level performances. In fact, in this case, $K_{p}$ did not increase (it remained almost null, providing transparency to the exoskeleton), and different joint trajectories with respect to the desired ones were performed. On the contrary, JBJ adaptation quickly brought the operator back to the desired coordination. $K_{p}$, with this strategy, increased fast to decrease joints errors. Finally, an in-between solution was provided by SJB adaptation, in which the $K_{p}$ increased but larger differences in the joint coordination were allowed. Figure 4 shows clearly that all the controllers allowed for similar end-effector error scores, thus the different inter-joint coordination did not affect the tracking task. 

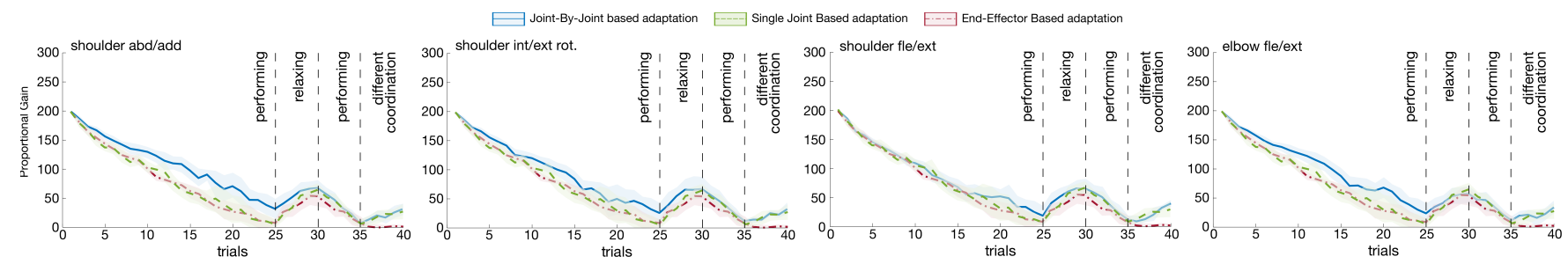

Fig. 3. Evolution of the four proportional gains $K_{p, i}$ during the experiment, averaged over the five subjects, for the three adaptive paradigms. At trial 35 , the gains were zeroed to have the same starting configuration for the last movements. For each condition, the shaded coloured area represents the standard error.
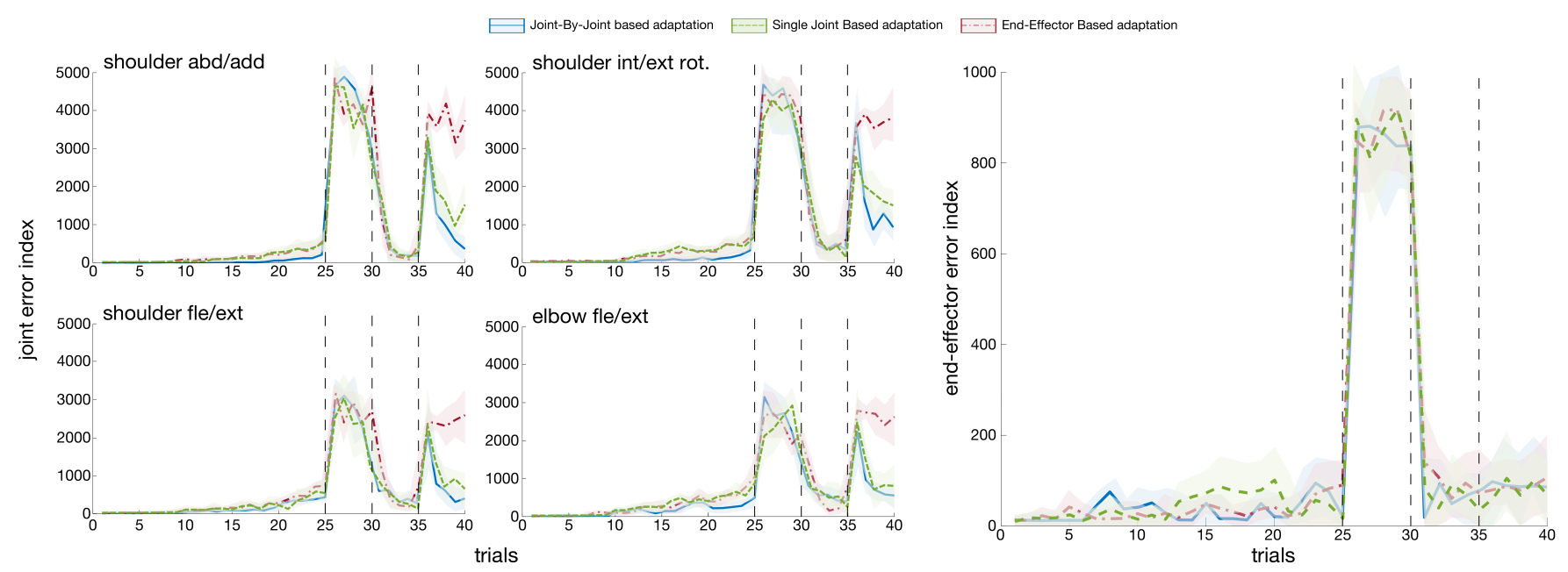

Fig. 4. On the left, average joint errors (sum of position and velocity error) and, on the right, average end-effector error (sum of position and velocity error), for the five subjects and the three adaptive processes. For each condition, the shaded coloured area represents the standard error.

\section{Discussion}

We determined three different adaptation strategies for our AAN controller, respectively based on the local error of each joint of the exoskeleton (JBJ), on the error of the end-effector (EEB), and on the error of a single joint (SJB), in particular the $\alpha_{H F}$ usually impaired in post-stroke survivors.

These three adaptation paradigms performed as expected in a typical tracking task, with variable voluntary behaviour of the human operators. All the protocols were able to react either to increased (trials 1-25, and 31-35) or to decreased activity (trials 25-30) by the subjects, showing the overall efficiency of the AAN control. Nonetheless it seems possible to suggest that a local adaptation strategy, as in JBJ, could better fit for early rehabilitation, when mostly targeting motor recovery. For example in this phase, as said, stroke survivors show decreased capacities to extend their elbow [19], having difficulties in achieving most of the motion tasks; a JBJ solution could provide better control along a desired reference coordination, performing better tracking of desired joint trajectories, while a global adaptation strategy (lke in EEB or SJB) could determine larger joint errors.

When considering instead the second part of the experiment (from trial 36), we targeted stroke survivors who are more capable of acting alone (late rehabilitation), a situation in which the controller should allow the patients to train intensively, with a minimal assistance to avoid negative behaviour.
EEB, due to its architecture, allowed any motion the subject wanted to perform (control gains remained almost null, keeping the robot in transparency, figure 3), as long as the end-effector task was achieved (small end-effector error in figure 4), and indeed it did not assist anymore the patient along the desired motion coordinations (large joint error in figure 4). Unfortunately it is clear that perfect performance at the end-effector may happen together with negative compensation at the shoulder level, producing potentially inadapt therapy [21]. We believe that adapting and basing the rehabilitation performance on a single parameter, as the end-effector score, could therefore in some case mislead the therapy and the robotic assistance.

Joint-by-joint assistance, on the contrary, once determined the desired joint trajectory, almost did not allow any different coordination. This paradigm, for later stages of stroke recovery, could be too strict. In fact, thanks to human arm redundancy, there may be slightly different coordination to complete the same task, without involving unnatural and pathological behaviour at the joint level or at the shoulder. Therefore the JBJ approach would also necessitate of highly customized references, since different patients may require different coordinations. One possible solution to use JBJ, even with chronic patients, would be to develop a simultaneous adaptation of the desired trajectories, in order to allow feasible and safe multiple strategies. But obviously these would increase the complexity of the controller and 


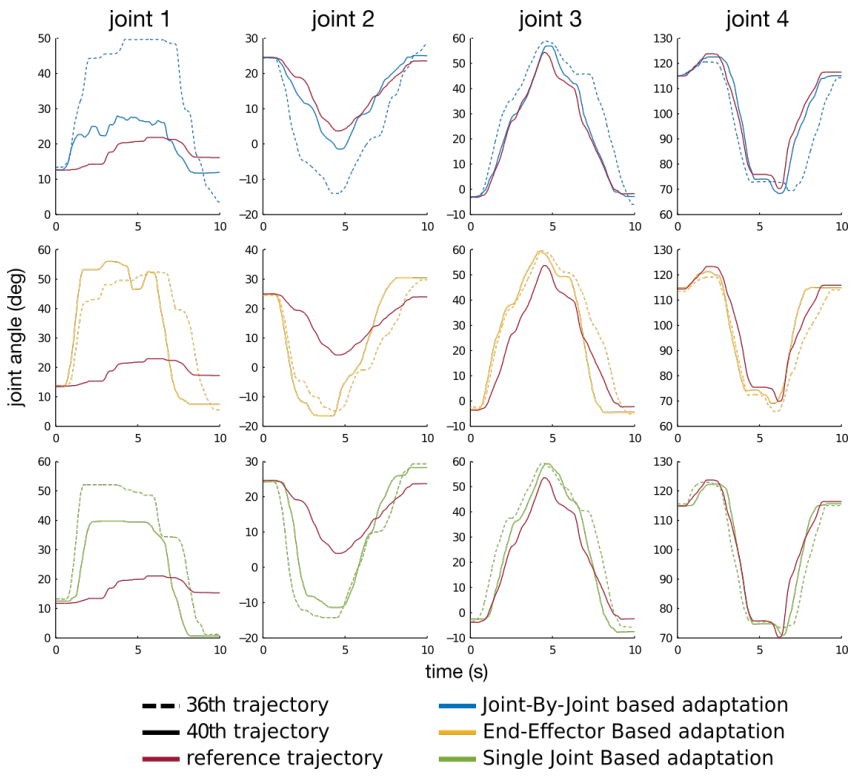

Fig. 5. Joint trajectories for the three strategies. Each column is one of the four joints of the ABLE exoskeleton. For each plot, three trajectories are shown: in red, the desired joint trajectory, dashed line the 36th motion (the first with a different inter-joint coordination), and in solid line the last motion (always with a different coordination, trial 40). First row is the JBJ adaptation, second EEB adaptation, third SJB adaptation.

would require a difficult online evaluation of the feasability of a coordination strategy.

Finally, the SJB adaptation seems to be promising. It better performed during the last trials, showing capabilities to assist the subject while leaving some freedom in slightly changing of the arm coordination. A weakness of this strategy is the global adaptation of the joint stiffness which generally produced larger joint errors during trials 1-25 w.r.t. local adaptation. A possible solution could be obtained by tuning the values of the adaptive parameters $(\beta$ and $\gamma$ ) differently for each joint, thus creating distinct joint adaptations to the same error signal. Of course, a drawback would be to determine the metrics to perform the mentioned tuning.

Therefore it seems clear that there is no a single optimal strategy to drive the adaptation in AAN controllers, above all without considering the specificity of the involved patients. However, this not trivial parameter must be carefully kept in consideration and tuned for improving the efficiency of any robot-led therapy.

In the future, more experiments will need to be conducted in order to validate these preliminary results, obtained by simulating unnatural behaviours by healthy participants, to evaluate the performances in a real scenario, with adaptation of the robotic behaviour to the less regular impaired motor capabilities of real stroke patients.

\section{REFERENCES}

[1] D. Mozaffarian, E. J. Benjamin, A. S. Go, D. K. Arnett, M. J. Blaha, M. Cushman, S. R. Das, S. de Ferranti, J.-P. Després, H. J. Fullerton, et al., "Heart disease and stroke statistics 2016 update a report from the american heart association," Circulation, p. 350, 2015.
[2] C. J. Winstein and D. B. Kay, "Translating the science into practice: shaping rehabilitation practice to enhance recovery after brain damage," Progress in Brain Research, 2015.

[3] P. Maciejasz, J. Eschweiler, K. Gerlach-Hahn, A. Jansen-Troy, and S. Leonhardt, "A survey on robotic devices for upper limb rehabilitation," Journal of neuroengineering and rehabilitation, vol. 11, no. 1, p. 1, 2014.

[4] V. S. Huang and J. W. Krakauer, "Robotic neurorehabilitation: a computational motor learning perspective," Journal of NeuroEngineering and Rehabilitation, vol. 6, no. 1, p. 5, 2009.

[5] T. Proietti, V. Crocher, A. Roby-Brami, and N. Jarrassé, "Upperlimb robotic exoskeletons for neurorehabilitation: a review on control strategies," Reviews in Biomedical Engineering, 2016.

[6] D. Lynch, M. Ferraro, J. Krol, C. M. Trudell, P. Christos, and B. T. Volpe, "Continuous passive motion improves shoulder joint integrity following stroke," Clinical rehabilitation, vol. 19, no. 6, pp. 594-599, 2005.

[7] Y. Mao and S. K. Agrawal, "A cable driven upper arm exoskeleton for upper extremity rehabilitation," in Robotics and Automation (ICRA), 2011 IEEE International Conference on. IEEE, 2011, pp. 4163-4168.

[8] M. Guidali, A. Duschau-Wicke, S. Broggi, V. Klamroth-Marganska, T. Nef, and R. Riener, "A robotic system to train activities of daily living in a virtual environment," Medical and biological engineering and computing, vol. 49, no. 10, pp. 1213-1223, 2011.

[9] N. Hogan, H. I. Krebs, B. Rohrer, J. J. Palazzolo, et al., "Motions or muscles? some behavioral factors underlying robotic assistance of motor recovery," Journal of rehabilitation research and development, vol. 43, no. 5, p. 605, 2006.

[10] J. L. Patton and F. A. Mussa-Ivaldi, "Robot-assisted adaptive training: custom force fields for teaching movement patterns," Biomedical Engineering, IEEE Transactions on, vol. 51, no. 4, pp. 636-646, 2004.

[11] N. Hogan and H. I. Krebs, "Interactive robots for neuro-rehabilitation," Restorative neurology and neuroscience, vol. 22, no. 3-5, pp. 349-358, 2004.

[12] T. Proietti, N. Jarrasse, A. Roby-Brami, and G. Morel, "Adaptive control of a robotic exoskeleton for neurorehabilitation," in Neural Engineering (NER), 2015 7th International IEEE/EMBS Conference on. IEEE, 2015, pp. 803-806.

[13] S. Balasubramanian and J. He, "Adaptive control of a wearable exoskeleton for upper-extremity neurorehabilitation," Applied Bionics and Biomechanics, vol. 9, no. 1, pp. 99-115, 2012.

[14] A. Pehlivan, D. Losey, and M. OMalley, "Minimal assist-as-needed controller for upper limb robotic rehabilitation," Robotics, IEEE Transactions on, vol. 32, no. 1, pp. 113-124, Feb 2016.

[15] R. Wei, S. Balasubramanian, L. Xu, and J. He, "Adaptive iterative learning control design for rupert iv," in Biomedical Robotics and Biomechatronics, 2008. BioRob 2008. 2nd IEEE RAS and EMBS International Conference on. IEEE, 2008, pp. 647-652.

[16] E. T. Wolbrecht, V. Chan, D. J. Reinkensmeyer, and J. E. Bobrow, "Optimizing compliant, model-based robotic assistance to promote neurorehabilitation," Neural Systems and Rehabilitation Engineering, IEEE Transactions on, vol. 16, no. 3, pp. 286-297, 2008.

[17] G. Ganesh, N. Jarrassé, S. Haddadin, A. Albu-Schaeffer, and E. Burdet, "A versatile biomimetic controller for contact tooling and haptic exploration," in Robotics and Automation (ICRA), 2012 IEEE International Conference on. IEEE, 2012, pp. 3329-3334.

[18] G. Wu, S. Siegler, P. Allard, C. Kirtley, A. Leardini, D. Rosenbaum, M. Whittle, D. D DLima, L. Cristofolini, H. Witte, et al., "Isb recommendation on definitions of joint coordinate system of various joints for the reporting of human joint motionpart i: ankle, hip, and spine," Journal of biomechanics, vol. 35, no. 4, pp. 543-548, 2002.

[19] A. Roby-Brami, A. Feydy, M. Combeaud, E. Biryukova, B. Bussel, and M. Levin, "Motor compensation and recovery for reaching in stroke patients," Acta neurologica scandinavica, vol. 107, no. 5, pp. 369-381, 2003.

[20] P. Garrec, J. P. Friconneau, Y. Measson, and Y. Perrot, "Able an innovative transparent exoskeleton for the upper-limb," in Intelligent Robots and Systems, 2008. IROS 2008. IEEE/RSJ International Conference on. IEEE, 2008, pp. 1483-1488.

[21] N. Nordin, S. Xie, and B. Wünsche, "Assessment of movement quality in robot-assisted upper limb rehabilitation after stroke: a review," JNER, vol. 11, no. 1, p. 137, 2014. 